\title{
A q-deformed Quantum Mechanics
}

\author{
Jian-zu Zhang ${ }^{a, b, c,}$
}

a) Max-Planck-Institut für Physik (Werner-Heisenberg Institut), Föhringer Ring 6, D-80805 München, Germany

b) Theoretische Physik, Universität Kaiserslautern, PO Box 3049, D-67653 Kaiserslautern, Germany

c) Institute for Theoretical Physics, Box 316, East China University of Science and Technology, 130 Mei Long Road, Shanghai 200237, P.R.China

With a q-deformed quantum mechanical framework, features of the uncertainty relation and a novel formulation of the Schrödinger equation are considered.

PACS: 03.65.Bz 
In searching for possible new physics at short distances (or high energy scale) consideration of the space structure is a useful guide. Quantum groups are a generalization of symmetry groups which have been used successfully in physics. A general feature of spaces carrying a quantum group structure is that they are noncommutative and inherit a well-defined mathematical structure from quantum group symmetries. In applications in physics, questions arise whether the structure can be used for physics at short distances and what phenomena could be linked to it. Recently, starting from such a noncommutative space as configuration space a generalization to a phase space is obtained [1]. This noncommutative phase space is derived from the noncommutative differential structure on configuration space [2]. Such noncommutative phase space is a $q$-deformation of the quantum mechanical phase space and thus all the machinery used in quantum mechanics can be applied in $q$-deformed quantum mechanics $[1,3,4,5]$.

In this letter we discuss the essential new features of a $q$-deformed quantum mechanics: (i) A q-deformed uncertainty relation: Here We find that the lowest limit of the Heisenberg uncertainty is undercut. (ii) A q-deformed dynamical equation which is found to be non-linear. The perturbative expansion of the later shows complex structure. In the lowest order approximation this equation is just the Schrödinger equation. The characteristics of the new equation are essentially non-perturbative. The qualitative behavior of its non-perturbative solutions is different from that of the Schrödinger equation. For example, the spectrum of the $q$-deformed harmonic oscillator is exponentially spaced [5].

\section{A $q$-deformed uncertainty relation}

The starting point of our investigation is the following $q$-deformed Heisenberg algebra $[1,6]$ :

$$
\begin{gathered}
q^{1 / 2} X P-q^{-1 / 2} P X=i U \\
U X=q^{-1} X U, \quad U P=q P U
\end{gathered}
$$

with the conjugation properties

$$
P^{\dagger}=P, \quad X^{\dagger}=X, \quad U^{\dagger}=U^{-1} .
$$


This $q$-deformed algebra is derived from the noncommutative differential structure on configuration space [2]. However, if $X$ is assumed to be a hermitean operator in a Hilbert space, the usual quantization rule $p \rightarrow-i \partial_{X}$ does not yield a hermitean momentum operator [1]. In order to define conjugation of $\partial_{X}$, and then a hermitean momentum operator $P$, it is necessary to introduce a unitary scaling operator $U$ satisfying (2) ${ }^{1}$. In Eqs. (1) and (2) the parameter $q$ is real and $q>1$. For the case of $q=1$, the scaling operator $U$ is reduced to a unit operator, and Eq.(1) reduces to the Heisenberg algebra. The non-trivial properties of the operator $U$ lead to a richer structure of algebra (1) and (2) than the Heisenberg algebra. From (1) and (3) we obtain

$$
X P-P X=i C
$$

where

$$
C=\left(U+U^{-1}\right) /\left(q^{1 / 2}+q^{-1 / 2}\right) .
$$

In order to show the special characteristics of the $q$-deformed uncertainty relation, we prove the following lemma.

Lemma. In any state the expectation value of the operator $C$ in (5) satisfies

$$
|<C>| \leq 1
$$

We note that $\left|<U+U^{-1}>\right| \leq 2$. Because $q^{1 / 2}+q^{-1 / 2} \geq 2$ for any $q>0$, we obtain lemma (6).

Equation (4) gives

$$
\Delta X \cdot \Delta P \geq \frac{1}{2}|<C>| .
$$

where $\Delta A=\sqrt{\left\langle(A-<A>)^{2}\right\rangle}$. Lema (6) shows that the lowest limit of the Heisenberg uncertainty relation $\Delta X \cdot \Delta P=\frac{1}{2}$ is undercut. It is interesting to show [7] that for the irreducible eigenstate $|n, \sigma>| s>$ of $P$ [1],

\footnotetext{
${ }^{1}$ The definitions of the scaling operator $U$, the conjugate $\bar{\partial}_{X}$ and $\partial_{X}$ and the hermitean momentum operator $P$ are [1]: $U^{-1}=q^{1 / 2}\left[1+(q-1) X \partial_{X}\right], \quad \bar{\partial}_{X}=-q^{-1 / 2} U \partial_{X}, \quad P=$ $-\frac{i}{2}\left(\partial_{X}-\bar{\partial}_{X}\right)$.
} 
$P|n, \sigma>| s>=P_{0}|n, \sigma>| s>$ with $P_{0}=\sigma s q^{n},(\sigma= \pm 1 ; 0<s<1 ; n=$ $0,1,2, \ldots)$, we have $\Delta P=0$, but $\Delta X$ is still finite. In fact, using (1)-(3), we obtain $\Delta X=\left(q-q^{-1}\right)^{-1}\left(q+q^{-1}\right)^{1 / 2} P_{0}^{-1}$. Thus we conclude that in the state $|n, \sigma>| s>, \Delta X \Delta P=0$. This is a surprising qualitative deviation from Heisenberg's uncertainty relation. It therefore raises the question where or not the conventional uncertainty relation is recovered at large scales. Unfortunately because of the complicated relations among $X, P$ and $U$, an explicit form of the right-hand side of the uncertainty relation as a function of $\Delta X$ and $\Delta P$ is not obtained at this stage. Thus when $q$ is some fixed value not equal to one it is still an important open question whether this $q$-deformed quantum mechanics does at all reproduce ordinary quantum mechanics at large scales. (7) may prove to be an important result within this formulation of a $q$-deformed quantum mechanics. Perhaps insights of a possible new physics just come from here.

The issue of uncertainty relations in the context of quantum group symmetric Heisenberg algebras was first considered by Kempf [8].

\section{The $q$-deformed dynamical equation}

The variables of the $q$-deformed algebra (1) and (2) can also be expressed in terms of the variables of an undeformed algebra. There are three pairs of canonical conjugate variables [1]: (i) The variables $\hat{x}, \hat{p}$ of the undeformed quantum mechanics; they satisfy $[\hat{x}, \hat{p}]=i$. (ii) The variables $\tilde{x}, \tilde{p}$; which are obtained by canonical transformation of $\hat{x}$ and $\hat{p}: \tilde{p}=f(\hat{z}) \hat{p}, \quad \tilde{x}=\hat{x} f^{-1}(\hat{z})$ where

$$
f^{-1}(\hat{z})=\frac{[\hat{z}-1 / 2]}{\hat{z}-1 / 2}, \quad \hat{z}=-\frac{i}{2}(\hat{x} \hat{p}+\hat{p} \hat{x})
$$

and $[A]=\left(q^{A}-q^{-A}\right) /\left(q-q^{-1}\right)$ for any $A$. The variables $\tilde{x}, \tilde{p}$ also satisfy $[\tilde{x}, \hat{p}]=i$. (iii) The $q$-deformed variables $X$ and $P$ where $X, P$ and the scaling operator $U$ are related to $\tilde{x}$ and $\tilde{p}$ in the following way:

$$
P=f^{-1}(\tilde{z}) \tilde{p}, \quad X=\tilde{x}, \quad U=q^{\tilde{z}}
$$

in $(9) \tilde{z}$ and $f^{-1}(\tilde{z})$ are defined by the same equations (8) for $\hat{z}$ and $f^{-1}(\hat{z})$. It is easy to check that $X, P$ and $U$ in (9) satisfy (1)-(3). 
our starting point is to use the $q$-deformed variables $X$ and $P$ to write down the Hamiltonian, then using (9) to represent $X$ and $P$ by $\tilde{x}$ and $\tilde{p}$. Because of $[\tilde{x}, \tilde{p}]=i$, (thus in the $\tilde{x}$ representation $\tilde{p}=-i \tilde{\partial}$, where $\tilde{\partial}=$ $\partial / \partial \tilde{x})$ all the machinery of quantum mechanics can be used for the $(\tilde{x}, \tilde{p})$ system. The $q$-deformed Hamiltonian of the system with potential $V(X)$ is $H(X, P)=P^{2} / 2 m+V(X)$. Using (1)-(3) and (9) the stationary dynamical equation of $q$-deformed quantum mechanics reads as

$$
\begin{gathered}
\left\{-\frac{1}{2 m}\left(q-q^{-1}\right)^{-2} \tilde{x}^{-2}\left[q\left(q^{-2 \tilde{x} \tilde{\partial}}-1\right)+q^{-1}\left(q^{2 \tilde{x} \tilde{\partial}}-1\right)\right]\right. \\
+V(\tilde{x})\} \psi(\tilde{x})=E_{q} \psi(\tilde{x})
\end{gathered}
$$

Eq. (10) is a non-linear equation which is a q-generalisation of the Schrödinger equation.

For the case $q$ is close to 1 , we let $q=e^{f}, 0<f \ll 1$. The perturbative expansion of the Hamiltonian is then

$$
\begin{aligned}
H= & -\frac{1}{2 m}\left(2 f+\frac{1}{3} f^{3}+\ldots\right)^{-2} \tilde{x}^{-2}\left[4 f^{2} \tilde{x}^{2} \tilde{\partial}^{2}+\frac{1}{3} f^{4}\left(4 \tilde{x}^{4} \tilde{\partial}^{4}\right.\right. \\
& \left.\left.+16 \tilde{x}^{3} \tilde{\partial}^{3}+10 \tilde{x}^{2} \tilde{\partial}^{2}\right)+\ldots\right]+V(\tilde{x}) .
\end{aligned}
$$

Thus to the lowest order in $f$, Eq. (10) reduces

$$
\left[-\frac{1}{2 m} \tilde{\partial}^{2}+V(\tilde{x})\right] \psi(\tilde{x})=E \psi(\tilde{x}) .
$$

This is just the Schrödinger equation for the $(\tilde{x}, \tilde{p})$ system. In (11) the next order correction of $H$ shows a complex structure which amounts to some additional momentum dependent interaction.

In the above we constructed the $q$-deformed Hamiltonian of the variables $X$ and $P$ in analogy with the undeformed system. Another possible way to construct a $q$-deformed Hamiltonian is that the system should act in accordance with a special algebra. An example is a $q$-deformed harmonic oscillator $[5]$.

If $q$-deformed quantum mechanics is a correct theory, its corrections to the undeformed theories must be very small at the energy range which can be reached by present-day experiments. In view of the present accuracy of 
tests of quantum electrodynamics at least down to $10^{-17} \mathrm{~cm}$, the effects of $q$-deformed quantum mechanics would show up at distances much smaller than $10^{-17} \mathrm{~cm}$. We hope that the $q$-deformed uncertainty relation might show some evidence in present-day experiments.

\section{Acknowledgements}

The author would like to thank Prof. J. Wess very much for giving the author the opportunity to join his project on quantum groups and for many stimulating helpful discussions and Prof. H. J. W. Müller-Kirsten for many helpful comments. He would also like to thank the Max-Planck-Institut für Physik (Werner-Heisenberg-Institut) for financial support and the Sektion Physik, Universität München, for warm hospitality. His work has also been supported by the Deutsche Forschungsgemeinschaft (Germany), the National Natural Science Foundation of China under Grant No. 19674014, and the Shanghai Education Development Foundation.

\section{References}

[1] M. Fichtmüller, A. Lorek, J. Wess, Z. Phys. C71 (1996) 533 .

[2] J. Wess, B. Zumino, Nucl. Phys.(Proc.Suppl.) 18B (1990) 302.

[3] J. Schwenk, J. Wess, Phys. Lett. B291 (1992) 273.

[4] A. Lorek, J. Wess, Z. Phys. C67 (1995) 671.

[5] A. Lorek, A. Ruffing, J. Wess, Z. Phys. C74 (1997) 369.

[6] A. Hebecker, S. Schreckenberg, J. Schwenk, W. Weich, J. Wess, Z. Phys. C64 (1994) 355.

[7] J. Wess, private conversation.

[8] A. Kempf, J. Math. Phys. 35 (1994) 4483. 